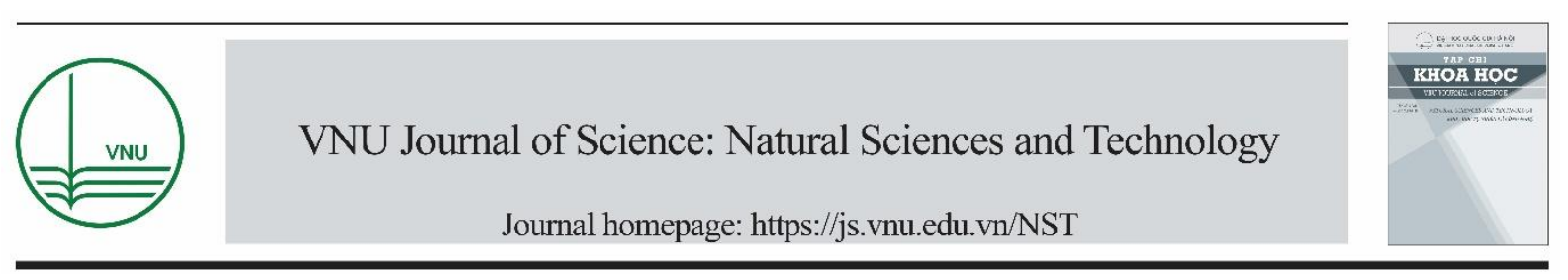

\title{
Original Article \\ The Effects of TDI on Selected Properties of Ester Epoxy Alkyd Varnish
}

\author{
Nguyen Trung Thanh* \\ Institute of Technology, General Department of National Defence Industry, \\ 3 Cau Vong, Bac Tu Liem, Hanoi, Vietnam
}

Received 30 July 2019

Revised 05 August 2020; Accepted 22 November 2020

\begin{abstract}
This article presents the effects of toluene diisocyanate (TDI) on electrical properties of ester epoxy alkyd varnish. Through testing on breakdown voltage, dielectric constant, block resistivity, Tang $\left(\mathrm{T}_{\mathrm{g}}\right)$ dielectric loss, the electrical properties of varnish were investigated. When TDI amount increased from to $4-10(\mathrm{w} / 100 \mathrm{w}$ of epoxy resin), the breakdown voltage increased from 21.78 to $65.69 \mathrm{KV} / \mathrm{mm}$. With TDI of $8-10 \mathrm{w} / \mathrm{w}$, the dielectric constant was the best, about $2.78-3.02$. With TDI of $8 \mathrm{w} / \mathrm{w}$, the block resistivity was $6.8 .10^{14} \Omega . \mathrm{cm}$ and $\mathrm{T}_{\mathrm{g}}$ dielectric loss was 0.0069 . The article also presents the effect of TDI on solvent- and heat resistance of the varnish.
\end{abstract}

Keywords: Ester epoxy alkyd v, electrical properties, solvent resistance, heat resistance.

\footnotetext{
*Corresponding author.

Email address: nguyentrungthanhk42@ gmail.com

https://doi.org/10.25073/2588-1140/vnunst.4933
} 


\title{
Nghiên cứu ảnh hưởng của chất đóng rắn TDI đến một số tính chất của vecny este epoxy alkyd
}

\author{
Nguyễn Trung Thành* \\ Viện Công nghệ, Tổng cuc Công nghiệp Quốc phòng, \\ 3 Cầu Vồng, Đúcc Thắng, Bắc Tùu Liêm, Hà Nội, Việt Nam \\ Nhận ngày 30 tháng 7 năm 2019 \\ Chỉnh sửa ngày 05 tháng 8 năm 2020; Chấp nhận đăng ngày 22 tháng 11 năm 2020
}

\begin{abstract}
Tóm tắt: Bài báo trình bày kết quả nghiên cứu ảnh hưởng của chất đóng rắn toluen diisoxyanat (TDI) đến tính chất điện của vecny este epoxy alkyd. Thông qua việc kiểm tra điện áp đánh thủng, hằng số điện môi, điện trở suất khối, Tang $\left(\mathrm{T}_{\mathrm{g}}\right)$ góc tổn hao điện môi, tính chất điện của màng sơn được khảo sát. Khi hàm lượng TDI tăng từ 4- 10 phần khối lượng, điện áp đánh thủng tăng từ 21,78 lên $65,69 \mathrm{KV} / \mathrm{mm}$. Với hàm lượng TDI từ $8-10$ phần khối lượng, sẽ có hằng số điện môi khoảng 2,78- 3,02. Với hàm lượng TDI 8 phần khối lượng điện trở suất khối là $6,8 \cdot 10^{14} \Omega$.cm và Tg góc tổn hao điện môi là 0,0069 . Bên cạnh đó, bài báo cũng giới thiệu kết quả nghiên cứu ảnh hưởng của chất đóng rắn TDI đến độ bền môi trường hóa chất, độ bền nhiệt của màng vecny este epoxy alkyd.
\end{abstract}

Tù khóa: Vecny este epoxy alkyd, tính chất điện, độ bền dung môi, độ bền nhiệt.

\section{Mở đầu}

Nhựa epoxy là loại nhựa nhiệt rắn được sử dụng rỗng rãi trong chế tạo màng phủ và keo dán do có khả năng bám dính tốt lên nhiều loại nền khác nhau,... [1]. Màng phủ từ nhựa epoxy có khả năng chịu ẩm và mù muối rất tốt, có khả năng chịu dung môi và hóa chất trong thời gian dài. Tính chất điện của epoxy trong môi trường ẩm $95-100 \%$ là ổn định ở dải nhiệt độ đến $150^{\circ} \mathrm{C}$ $[2,3]$. Các lợi thế này đã đưa epoxy lên hàng đầu những polyme được sử dụng nhiều trong kỹ thuật điện - điện tử [3]. Nhược điểm của nhựa epoxy là giòn, điều này có thể giải quyết bằng cách sử dụng các chất hóa dẻo, hoặc chọn chất đóng rắn thích hợp hoặc biến tính với nhựa alkyd, cao su, thiokol, oligome acrylate,... [4-8]. Một số tác giả đã sử dụng epoxy được hóa dẻo bằng cách biến tính với nhựa alkyd (este hóa nhựa epoxy bằng

\footnotetext{
*Tác giả liên hệ.

Địa chỉ email: nguyentrungthanhk42@gmail.com

https://doi.org/10.25073/2588-1140/vnunst.4933
}

nhựa alkyd) để làm sơn và khảo sát thời gian khô của màng vecny, độ cứng, độ bóng, độ bền uốn, khả năng chịu nhiệt,... [9]. Do vecny este epoxy alkyd kết hợp được những ưu điểm của nhựa epoxy như bám dính tốt, bền môi trường dung môi, cách điện cao,... và độ mềm dẻo của nhựa alkyd, kết quả nghiên cứu nhằm đánh giá khả năng sử dụng vecny này cho các chi tiết, linh kiện điện tử trong một số môi trường hóa chất. Bài báo này trình bày ảnh hưởng của tỷ lệ đóng rắn toluen diisoxyanat (TDI) đến điện áp đánh thủng, hằng số điện môi, điện trở suất khối, Tang $(\mathrm{Tg})$ góc tổn hao điện môi của vecny este epoxy alkyd. Bên cạnh đó, bài báo cũng đưa ra kết quả khảo sát ảnh hưởng của tỷ lệ chất đóng rắn TDI đến độ bền trong môi trường xăng, dầu nhờn, axit clohydric, muối ăn và khảo sát độ bền nhiệt của màng veny este epoxy alkyd. 


\section{Thực nghiệm}

\subsection{Nguyên liệu và hóa chất}

- Nhựa este epoxy alkyd mac E-30 (Nga), hàm rắn $60 \%$, hàm lượng nhóm epoxy $13-15$.

- Chất đóng rắn TDI, tên thương mại Desmodur L 75 của hãng Bayer (Đức), dung dịch 75\% toluen-2,4-diisoxyanat (TDI) trong dung môi etyl acxetat, hàm lượng nhóm $\mathrm{NCO}: 13,3$ và tỷ trọng 1,17.

- Axeton, chất hóa dẻo Dioctyl Phtalat (DOP), xylen, butyl axetat: công nghiệp (Trung Quốc).

\subsection{Chế tạo mẫu}

- Chuẩn bị nguyên liệu.

- Cân nguyên liệu theo đơn nghiên cứu. Hòa tan este epoxy alkyd trong hỗn hợp dung môi axeton, xylen, butyl axetat để hàm rắn đạt $30 \%$, bổ sung $3 \%$ chất hóa dẻo DOP.

- Rót chất đóng rắn TDI vào dung dịch vecny este epoxy alkyd theo tỷ lệ đã tính toán trước. Khuấy đều mẫu vecny đã pha đóng rắn và gia công mẫu (tiêu chuẩn TCVN 2090:2007) trên tấm đồng kích thước 100x100x1mm để kiểm tra tính chất điện, độ bền môi trường hóa chất.

\subsection{Phương pháp thử nghiệm đánh giá}

- Điện trở suất khối được đo trên thiết bị R8340A hãng ADVANTEST (Nhật Bản) xác định theo TCVN 7918:2008.

- Điện áp đánh thủng được đo trên thiết bị TOS9021 hãng KIKUSUI (Nhật Bản) xác định theo TCVN 9630-1:2013.

- Tg góc tổn hao điện môi, hằng số điện môi được đo trên thiết bị đo thông số điện môi Quadtech 7600 (Mỹ). Tg góc tổn hao điện môi, hằng số điện môi xác định theo TCVN 98902:2013 với tần số $10^{6} \mathrm{~Hz}$. Các tính điện được đo tại Tổng cục Tiêu chuẩn- Đo lường chất lượng.

- Độ bền nhiệt: Phân tích nhiệt khối lượng (TGA) được thực hiện trên thiết bị NETZSCH TG 209F1 LIBRA tại Viện Kỹ thuật nhiệt đớiViện Hàn lâm Khoa học và Công nghệ Việt Nam. Điều kiện đo trong khí nitơ với tốc độ nâng nhiệt $10^{\circ} \mathrm{C} /$ phút từ nhiệt độ phòng đến $600^{\circ} \mathrm{C}$.

- Thử nghiệm sự thay đổi khối lượng của màng vecny trong môi trường xăng $\mathrm{A} 95$, thử nghiệm khả năng chịu môi trường hóa chất của màng vecny theo tiêu chuẩn TCVN 105171:2014 ở điều kiện nhiệt độ $(23 \pm 2)^{\circ} \mathrm{C}$ và độ ẩm tương đối $(50 \pm 5) \%$ tại Viện Công nghệ- Tổng cục Công nghiệp Quốc phòng.

\section{Kết quả và thảo luận}

\subsection{Nghiên cứu ảnh huởng của hàm luợng TDI đến tính chất điện của màng vecny}

3.1.1. Nghiên cúu ảnh huởng của hàm luợng TDI đến điện áp đánh thủng của màng vecny

Để nghiên cứu ảnh hưởng của hàm lượng TDI đến điện áp đánh thủng của màng vecny trên cơ sở nhựa este epoxy alkyd (EEP), các mẫu vecny được chế tạo với hàm lượng TDI $4 ; 6 ; 8$; 10; 12 phần khối lượng (PKL). Các mẫu vecny sau khi chế tạo được gia công trên các tấm mẫu đồng tiêu chuẩn. Kết quả nghiên cứu được thể hiện trong Bảng 1.

Bảng 1. Ảnh hưởng của hàm lượng TDI đến điện áp đánh thủng màng vecny

\begin{tabular}{|c|c|c|c|}
\hline \multirow{2}{*}{ Tên mẫu } & \multicolumn{2}{|c|}{$\begin{array}{c}\text { Tỷ lệ vecny } \\
\text { EEP/TDI (PKL) }\end{array}$} & \multirow{2}{*}{$\begin{array}{c}\text { Piện áp đánh thủng } \\
(\mathrm{kV} / \mathrm{mm})\end{array}$} \\
\cline { 2 - 3 } & EEP & TDI & \\
\hline M1 & 100 & 4 & 21,78 \\
\hline M2 & 100 & 6 & 23,05 \\
\hline M3 & 100 & 8 & 39,76 \\
\hline M4 & 100 & 10 & 65,69 \\
\hline M5 & 100 & 12 & 56,64 \\
\hline
\end{tabular}

Từ Bảng 1 nhận thấy, tăng lượng chất đóng rắn $\mathrm{TDI}$ thì điện áp đánh thủng tăng lên. Với hàm lượng TDI từ 4 đến 10 PKL thì điện áp đánh thủng tăng từ 21,78 lên đến $65,69 \mathrm{kV} / \mathrm{mm}$, tuy nhiên, tiếp tục tăng hàm lượng TDI thì giá trị này lại giảm xuống. Điều này được giải thích, điện áp đánh thủng là quá trình tăng số lượng electron trong chất điện môi dưới ảnh hưởng của điện trường mạnh. Các quá trình này gồm: Sự ion hoá do va đập electron lên nguyên tử hoặc ion trong mạng lưới, sự tự phát xạ electron từ catot, tính đồng nhất của vật liệu hình dạng của điện trường,... [10]. Khi hàm lượng TDI vượt 10 PKL, lượng chất đóng rắn TDI bị dư, dẫn tới các nhóm chức NCO tự do, nhóm chức phân cực trong vật liệu xuất hiện với mật độ cao hơn làm 
cho vật liệu dễ bị ion hóa dưới điện trường mạnh, dẫn tới điện áp đánh thủng giảm xuống.

\subsubsection{Nghiên cứu ảnh hương của hàm luọng TDI đến hằng số điện môi của màng vecny}

Một trong những thông số đánh giá tính chất điện là giá trị hằng số điện môi, những chất có giá trị hằng số điện môi nhỏ sẽ cho giá trị cách điện tốt. Các mẫu vecny được khảo sát là $\mathrm{M} 1$, $\mathrm{M} 2, \mathrm{M} 3, \mathrm{M} 4, \mathrm{M} 5$ có tỷ lệ thành phần như đề cập ở Bảng 1. Kết quả nghiên cứu được thể hiện trong Bảng 2.

Bảng 2. Ảnh hưởng của hàm lượng TDI đến hằng số điện môi của màng vecny

\begin{tabular}{|c|c|c|}
\hline TT & Tên mẫu & Hằng số điện môi \\
\hline 1 & M1 & 4,32 \\
\hline 2 & M2 & 3,45 \\
\hline 3 & M3 & 3,02 \\
\hline 4 & M4 & 2,78 \\
\hline 5 & M5 & 3,64 \\
\hline
\end{tabular}

Kết quả Bảng 2 cho thấy, khi tăng hàm lượng TDI từ 4- 10 PKL thì hằng số điện môi giảm dần từ 4,32 xuống đến 2,78 . Tuy nhiên, khi hàm lượng TDI là $12 \mathrm{PKL}$ thì hằng số tổn hao điện môi lại tăng. Điều này được giải thích, phản ứng đóng rắn màng vecny $\mathrm{EEP}$ bằng $\mathrm{TDI}$ thông qua nhóm $\mathrm{OH}$ của epoxy và nhóm $\mathrm{NCO}$ của TDI, theo phản ứng:

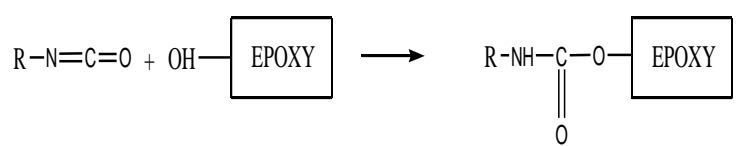

Với hàm lượng TDI tăng từ 4-10 PKL thì mật độ đan lưới trong vecny tăng lên, trong thành phần cấu tạo của màng vecny không còn dư nhiều các nhóm chức hoạt hóa, phân cực nên vecny gần như trung hòa về điện nên giá trị hằng số điện môi giảm dần. Khi tăng lượng TDI lên đến $12 \mathrm{PKL}$, trong thành phần vecny sẽ dư nhóm chức NCO dẫn tới vật liệu bị phân cực làm cho hằng số điện môi tăng lên [10].

\subsubsection{Nghiên cứu ảnh hưởng của hàm luợng TDI đến điện trở suất khối của màng vecny}

Các mẫu vecny được khảo sát là $\mathrm{M} 1, \mathrm{M} 2$, M3, M4, M5 có tỷ lệ thành phần như đề cập ở Bảng 1. Kết quả khảo sát được thể hiện trong Bảng 3.
Bảng 3. Ảnh hưởng của hàm lượng TDI đến điện trở suất khối của màng vecny

\begin{tabular}{|c|c|c|}
\hline TT & Tên mẫu & Điện trở suất khối $(\Omega . c m)$ \\
\hline 1 & M1 & $1,3.10^{14}$ \\
\hline 2 & M2 & $3,7.10^{14}$ \\
\hline 3 & M3 & $6,8.10^{14}$ \\
\hline 4 & M4 & $2,6.10^{14}$ \\
\hline 5 & M5 & $1,8.10^{14}$ \\
\hline
\end{tabular}

Kết quả Bảng 3 cho thấy, khi tăng hàm lượng TDI thì điện trở suất khối tăng từ $1,3 \cdot 10^{14} \Omega$.cm đối với mẫu có 4 PKL lên $6,8.10^{14} \Omega$.cm đối với mẫu sử dụng $8 \mathrm{PKL}$. Tuy nhiên, với hàm lượng TDI là 10 PKL thì điện trở suất khối lại giảm xuống còn $2,6.10^{14} \Omega . c m$. Điều này được giải thích, điện trở suất khối của màng vecny phụ thuộc vào độ linh động và sự có mặt của các điện tích tự do trong phân tử. Mặt khác, các tính chất điện của màng vecny chịu ảnh hưởng lớn của tạp chất, hàm lượng ẩm,... Đối với các mẫu có hàm lượng chất đóng rắn TDI cao hoặc thấp $(4,6,8$ PKL) sau khi vecny khô trong thành phần vẫn còn dư các thành phần este epoxy alkyd (với mẫu sử dụng hàm lượng TDI thấp) hoặc dư chất đóng rắn TDI (với mẫu sử dụng $10,12 \mathrm{PKL}$ ), đồng thời là việc còn dư các nhóm chức tự do tăng khả năng hút ẩm của màng vecny, điều này dẫn tới điện trở suất khối bị giảm [11].

3.1.4. Nghiên cứu ảnh hưởng của hàm lượng TDI đến $T g$ của góc tổn hao điện môi của màng vecny

Các mẫu vecny được khảo sát là $\mathrm{M} 1, \mathrm{M} 2$, M3, M4, M5 như đã đề cập ở Bảng 1. Kết quả khảo sát được thể hiện trong Bảng 4 .

Bảng 4. Ảnh hưởng của hàm lượng TDI đến Tg của góc tổn hao điện môi

\begin{tabular}{|c|c|c|}
\hline TT & Tên mẫu & Tg góc tổn hao điện môi \\
\hline 1 & M1 & 0,0351 \\
\hline 2 & M2 & 0,0092 \\
\hline 3 & M3 & 0,0069 \\
\hline 4 & M4 & 0,0078 \\
\hline 5 & M5 & 0,0090 \\
\hline
\end{tabular}

Bảng 4 cho thấy, khi tăng hàm lượng TDI thì $\mathrm{Tg}$ của góc tổn hao điện môi giảm dần từ 0,0351 đối với mẫu M1 xuống 0,0069 đối với mẫu M3. Tuy nhiên, với hàm lượng TDI là 10 và $12 \mathrm{PKL}$ 
thì Tg của góc tổn hao điện môi lại tăng lên. Tổn hao điện môi trên đơn vị thể tích vật liệu sẽ tỷ lệ thuận với tần số điện trường và $T g \delta$, với góc $\delta$ là góc chênh lệch pha giữa điện trường trong và ngoài vật liệu. Theo đặc điểm và bản chất vật lý, đối với dạng vật liệu vecny nghiên cứu, tổn hao điện môi chủ yếu là do phân cực [11]. Với các mẫu có hàm lượng TDI từ $4,6,8$ PKL, khi tăng hàm lượng TDI thì mức độ đan lưới của màng vecny tăng lên, các nhóm chức phân cực giảm theo phản ứng đan lưới, đồng thời vật liệu trở nên đồng nhất hơn (ở đây có phản ứng hóa học để liên kết hai thành phần tham gia phản ứng, tạo thành đại phân tử có cấu trúc mạng không gian). Khi hàm lượng TDI tăng lên đến 10,12 PKL, giá trị Tg của góc tổn hao điện môi lại tăng. Nguyên nhân có thể là do ở hàm lượng này, có một lượng TDI dư trong vecny làm cho mật độ nhóm chức NCO tăng, dẫn tới vật liệu bị tăng độ phân cực, vật liệu bị phân lớp, không đồng nhất.

3.2. Nghiên cứu ảnh hưởng của hàm lương TDI tới độ bền môi truờng hóa chất của màng vecny

3.2.1. Khảo sát sụ thay đổi khối lượng của màng vecny trong môi truờng xăng A95

Các mẫu khảo sát là M1, M2, M3, M4, M5 như đề cập ở Bảng 1 . Kết quả khảo sát được thể hiện trong Bảng 5.

Bảng 5. Sự thay đổi khối lượng mẫu theo thời gian ngâm trong xăng A95

\begin{tabular}{|c|c|c|c|c|c|}
\hline \multirow{2}{*}{ Thồng số } & \multicolumn{5}{|c|}{ Phần khối lượng } \\
\cline { 2 - 6 } & tăng theo thời gian ngâm mẫu, \% \\
\cline { 2 - 6 } & $\mathrm{M} 1$ & $\mathrm{M} 2$ & $\mathrm{M} 3$ & $\mathrm{M} 4$ & $\mathrm{M} 5$ \\
\hline 0 giờ & 0 & 0 & 0 & 0 & 0 \\
\hline 0,5 giờ & 15,65 & 10,15 & 6,15 & 4,15 & 3,10 \\
\hline 1 giờ & 19,58 & 11,89 & 7,89 & 5,89 & 3,89 \\
\hline 2 giờ & 22,48 & 12,76 & 8,76 & 6,76 & 4,76 \\
\hline 4 giờ & 24,16 & 13,45 & 9,45 & 7,45 & 4,95 \\
\hline
\end{tabular}

\begin{tabular}{|c|c|c|c|c|c|}
\hline 8 giờ & 22,75 & 12,58 & 8,58 & 7,08 & 4,58 \\
\hline 24 giờ & 21,46 & 12,26 & 7,90 & 6,60 & 4,26 \\
\hline 48 giờ & 20,50 & 12,01 & 7,61 & 6,36 & 4,01 \\
\hline 72 giờ & 20,51 & 11,85 & 7,60 & 6,05 & 3,85 \\
\hline 96 giờ & 20,53 & 11,77 & 7,47 & 5,97 & 3,77 \\
\hline 120 giờ & 20,54 & 11,78 & 7,48 & 5,98 & 3,78 \\
\hline
\end{tabular}

Kết quả nghiên cứu cho thấy, trong khoảng 30 phút đầu, mẫu có độ tăng khối lượng rất nhanh. Giai đoạn này, vecny bị trương nở trong xăng. Quá trình diễn ra nhanh trong khoảng 04 giờ đầu ngâm mẫu. Sau khoảng thời gian 08 giờ ngâm mẫu có hiện tượng giảm khối lượng. Việc giảm khối lượng mẫu có thể do este epoxy alkyd dư, các chất thấp phân tử, tạp chất... bị hòa tan. Sau khoảng thời gian ngâm mẫu 48 giờ, khối lượng mẫu ổn định. Hàm lượng chất đóng rắn TDI tăng thì vecny sẽ bền trong dung môi xăng A95 hơn. Điều này có thể được giải thích, khi tăng tỷ lệ chất đóng rắn thì mật độ đan lưới tăng làm cho mạch polyme hình thành cấu trúc không gian bền vững hơn làm cho polyme trở nên khó, thậm chí không thể hòa tan, cấu trúc không gian bền vững cũng làm cho dung môi rất khó khuyếch tán kéo dãn mạch polyme để làm tăng khối lượng và thể tích của polyme. Đối với các mẫu có dư chất đóng rắn M5 thì thành phần TDI cũng được tạo mạng không gian bởi kết với hơi ẩm, thành phần này rất bền vững với dung môi [12].

3.2.2. Khảo sát khả năng chịu môi truò̀ng hóa chất của màng vecny

Để nghiên đánh giá khả năng sử dụng trong một số môi trường khác nhau các mẫu vecny khảo sát là $\mathrm{M} 1, \mathrm{M} 2, \mathrm{M} 3, \mathrm{M} 4, \mathrm{M} 5$ có tỷ lệ thành phần như đề cập ở Bảng 1. Các khảo sát khả năng chịu môi trường hóa chất của màng vecny được tiến hành trong dầu nhờn Castrol Activ, axit $\mathrm{HCl}$ $5 \%, \mathrm{NaCl} 5 \%$. Kết quả khảo sát được trình bày trong Bảng 6.

Bảng 6. Khả năng chịu môi trường hóa chất của màng vecny

\begin{tabular}{|c|c|c|c|c|}
\hline \multirow{2}{*}{ TT } & \multirow{2}{*}{$\begin{array}{c}\text { Tên } \\
\text { mẫu }\end{array}$} & \multicolumn{3}{|c|}{ Bề mặt của màng vecny (thời gian ngâm mẫu 72 giờ) } \\
\cline { 3 - 5 } & Độ bền dầu nhờn (Castrol Activ) & Độ bền trong axit HCl 5\% & Độ bền trong NaCl 5\% \\
\hline 1 & M1 & Màng vecny bị phồng rộp & Màng vecny bị phồng rộp & Không đổi \\
\hline 2 & M2 & Không đồi & Màng vecny bi giảm độ bóng & Không đồi \\
\hline 3 & M3 & Không đồi & Không đổi & Không đồi \\
\hline 4 & M4 & Không đổi & Không đổi & Không đổi \\
\hline 5 & M5 & Không đổi & Không đổi & Không đổi \\
\hline
\end{tabular}


Kết quả nghiên cứu trong Bảng 6 cho thấy, các mẫu vecny M3, M4, M5 có độ bền cao đối với dầu nhờn, axit $\mathrm{HCl} 5 \%$ và $\mathrm{NaCl} 5 \%$, điều này cho thấy lựa chọn tỷ lệ chất đóng rắn TDI phù hợp thì màng vecny tạo ra có khả năng sử dụng để bảo vệ các chi tiết điện tử trong một số môi trường hóa chất.

\subsection{Nghiên cứu ảnh hưởng của hàm lượng TDI} đến độ bền nhiệt của màng vecny

Để nghiên cứu ảnh hưởng của tỷ lệ chất đóng rắn TDI đến độ bền nhiệt của màng vecny phương pháp phân tích nhiệt trọng lượng TGA được thực hiện. Các mẫu khảo sát là M1, M2, M3, M4, M5 có tỷ lệ thành phần như đề cập ở Bảng 1. Kết quả nghiên cứu được thể hiện trong Bảng 7 và Hình $1 \mathrm{a}, 1 \mathrm{~b}, 1 \mathrm{c}, 1 \mathrm{~d}, 1 \mathrm{e}$.

Bảng 7. Ảnh hưởng của hàm lượng TDI tới độ bền nhiệt của màng vecny

\begin{tabular}{|c|c|c|}
\hline \multirow{2}{*}{$\begin{array}{c}\text { Ký } \\
\text { hiệu } \\
\text { mấu }\end{array}$} & \multicolumn{2}{|c|}{ Khối lượng phân hủy $(\%)$} \\
\cline { 2 - 3 } & $\begin{array}{c}\text { Từ nhiệt độ phòng } \\
\text { đên } 320^{\circ} \mathrm{C}\end{array}$ & $\begin{array}{c}\text { Từ nhiệt độ phòng } \\
\text { đên } 600^{\circ} \mathrm{C}\end{array}$ \\
\hline M1 & 21,72 & 84,43 \\
\hline M2 & 19,90 & 78,23 \\
\hline M3 & 19,92 & 77,55 \\
\hline M4 & 18,02 & 77,52 \\
\hline M5 & 17,12 & 71,00 \\
\hline
\end{tabular}

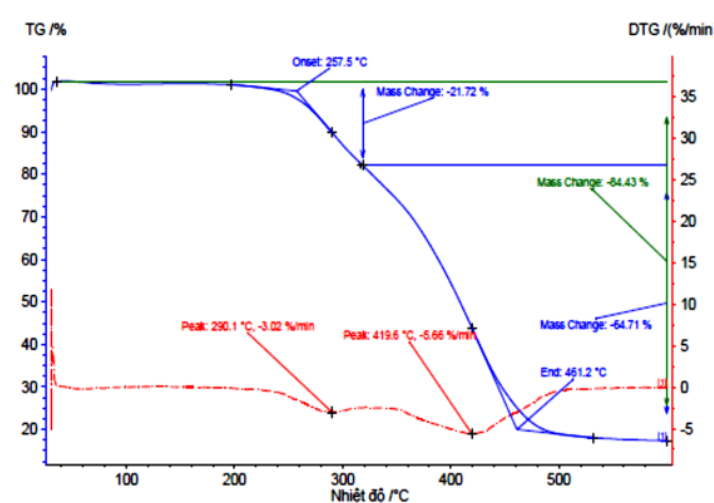

Hình 1a. Giản đồ TGA của mẫu M1.

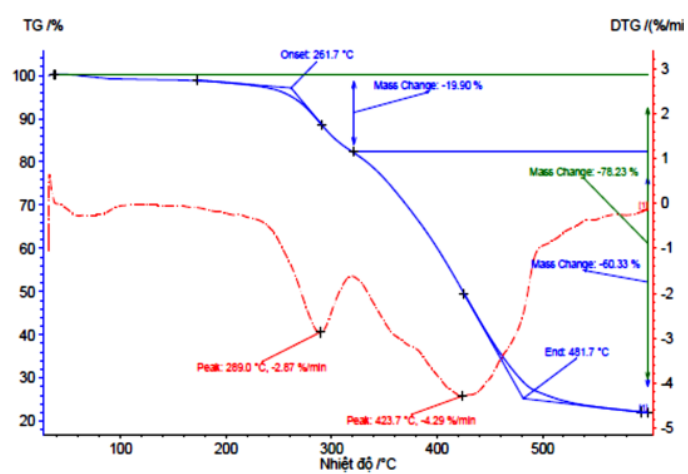

Hình 1b. Giản đồ TGA của mẫu M2.

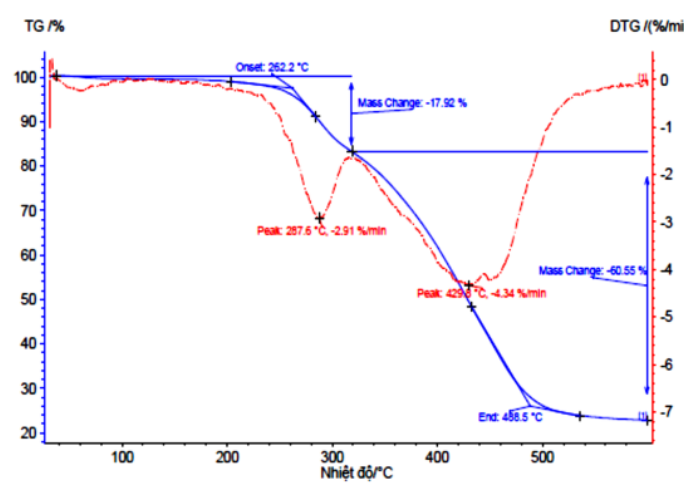

Hình 1c. Giản đồ TGA của mẫu M3.

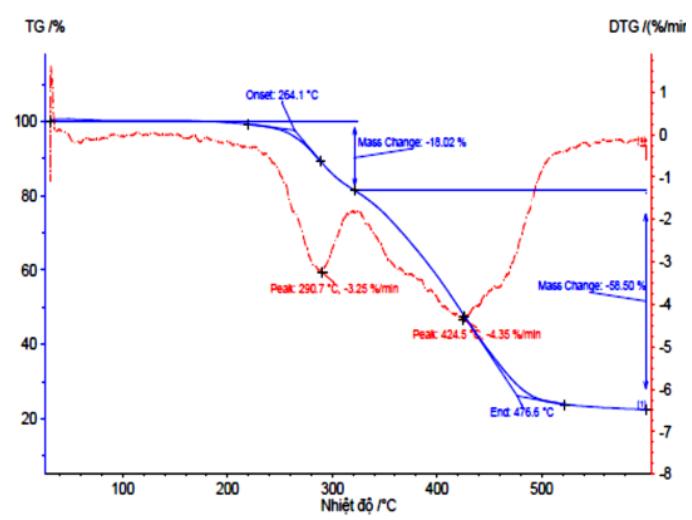

Hình 1d. Giản đồ TGA của mẫu M4. 


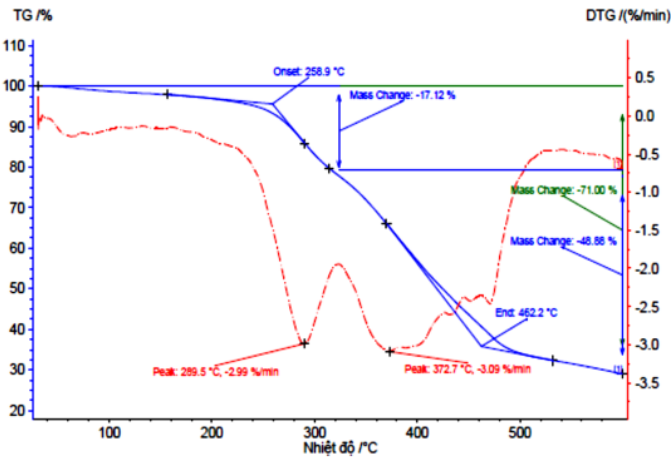

Hình 1e. Giản đồ TGA của mẫu M5.

Từ kết quả Bảng 7 và Hình $1 \mathrm{a}, 1 \mathrm{~b}, 1 \mathrm{c}, 1 \mathrm{~d}$, 1e cho thấy, trong khoảng nhiệt độ dưới $200^{\circ} \mathrm{C}$ : là giai đoạn phân hủy của các chất thấp phân tử, dung môi dư. Lượng phân hủy khoảng $2-7 \%$. Trong khoảng nhiệt độ từ nhiệt độ phòng đến $320^{\circ} \mathrm{C}$ : Là giai đoạn phân hủy của nhóm chức còn dư trong mạch polyme, các chất thấp phân tử,... Giai đoạn này cho thấy: Mẫu M1 có độ phân hủy là 21,72, lớn nhất. Mẫu M5 có độ phân hủy là 17,12 , các mẫu M2, M3, M4 mức độ phân hủy chênh lệch nhau không nhiều, từ 17,92-19,90\%. Tổng khối lượng phân hủy từ nhiệt độ phòng đến $600^{\circ} \mathrm{C}$, mầu $\mathrm{M} 1$ có mức độ phân hủy cao nhất, 84,43\%. Mẫu M5 có mức độ phân hủy thấp nhất, $71 \%$. Các mẫu M2, M3, M4 mức độ phân hủy gần như tương đương nhau, từ 77,52$78,23 \%$. Như vậy, có thể khẳng định, với tỷ lệ chất đóng rắn TDI từ $6-10 \mathrm{PKL}$, mức độ tạo mạng không gian tương đương nhau.

\section{Kết luận}

- Tăng hàm lượng TDI từ 4 - 10 PKL thì điện áp đánh thủng tăng từ 21,78 lên $65,69 \mathrm{KV} / \mathrm{mm}$, khi hàm lượng TDI là $12 \mathrm{PKL}$ thì giá trị này là $56,65 \mathrm{KV} / \mathrm{mm}$.

- Khi sử dụng TDI từ 4 - 10 PKL sẽ giảm giá trị hằng số điện môi. Hằng số điện môi giảm từ đạt giá trị thấp nhất là 2,78 khi hàm lượng TDI là $10 \mathrm{PKL}$.

- Điện trở suất khối đạt 6,8.1014 $\Omega . c m$ khi sử dụng lượng TDI là 8 PKL.
- Hàm lượng chất đóng rắn TDI từ $8-10$ PKL sẽ làm giảm giá trị $T g$ của góc tổn hao điện môi. $\mathrm{Tg}$ của góc tổn hao điện môi đạt 0,0069 0,0078 .

- Tăng hàm lượng chất đóng rắn TDI độ bền môi trường, độ bền nhiệt của vecny tăng lên. Tuy nhiên, khi tăng tới một tỷ lệ lớn hơn 10 PKL thì tính chất điện lại bị giảm đi.

\section{Tài liệu tham khảo}

[1] B.J. Rohde, M.L. Robertson, Ramanan Krishnamoorti, Concurrent curing kinetics of an anhydride-cured epoxy resin and polydicyclopentadiene, Polymer 69 (2015) 204- 214.

[2] P.G. Vu, T.T.X. Hang, V.K. Oanh, T.A. Truc, T.T. Thuy, Study on anti-corrosion protection ability of epoxy coating CNT/ZnO-clay nanocomposite, Vietnam Journal of Chemistry 55 (2017) 308-312. https://doi.org/ 10.15625/08667144.2017-00464 (In Vietnamese).

[3] M. Palimi, E. Alibakhshi, G. Bahlakeh, B. Ramezanzadeh, Electrochemical investigations of the corrosion protection properties of an epoxyester coating filled with cerium acetyl acetonate anticorrosive pigment, Electrochemical Society 164 (2017) 709-716.

[4] G.M. Roudsari, A.K. Mohanty, M. Misra, Study of the Curing Kinetics of Epoxy Resins with Biobased Hardener and Epoxidized Soybean Oil, ACS Sustainable Chemical Engineering 9 (2014) 2111-2116.

[5] P. Czub, I. Franek, Epoxy resins modified with palm oil derivatives preparation and properties, Polimery 58 (2013) 135-139.

[6] R. Jack, A.U. Anya, O.F. Osagie, Comparative Studies of Oil-Modified Alkyd Resins Synthesized from Epoxidized and Crude Neem Oil, American Journal of Applied Chemistry 4 (2016) 120-124.

[7] N. Saba, M. Jawaid, O.Y Alothman, P.M. Tahir, Recent advances in epoxy resin, natural fiberreinforced epoxy composites and their applications, Journal of Reinforced Plastics and Composites 35 (2016) 447-470.https://doi.org/10. 1177/0731684415618459.

[8] P. Gogoi, M. Boruah, S. Sharma, S.K. Dolui, Blends of Epoxidized Alkyd Resins Based on Jatropha Oil and the Epoxidized Oil Cured with Aqueous Citric Acid Solution: A Green Technology Approach. ACS Sustainable Chemical Engineering 2 (2015) 261-268. 
[9] S. Morsch, Y. Liu, P. Greensmith, S.B. Lyon, S.R. Gibbon, Molecularly controlled epoxy network nanostructures, Polymer 108 (2017) 146153.

[10] F. Mustata, N. Tudorachi, D. Rosu, Curing and Thermal Behavior of Resin Matrix for Composites Based on Epoxidized Soybean Oil/Diglycidyl Ether of Bisphenol A, Engineering 42 (2011) 1803-1812.
[11] J. Zhang, S. Hu, G. Zhan, X.Tang, Y. Yu, Biobasednanocomposites from clay modified blend of epoxidized soybean oil and cyanate ester resin, Progress in Organic Coatings 76 (2013) 1683-1690.

[12] A. Sienkiewicz, P. Czub, Novel bio-based epoxypolyurethane materials from modified vegetable oils - synthesis and characterization, eXPRESS Polymer Letters 11 (2017) 308-319. 